Research Paper

\title{
Right Ventricular and Right Atrial Involvement Can Predict Atrial Fibrillation in Patients with Hypertrophic Cardiomyopathy?
}

\author{
Christina Doesch ${ }^{1,2}{ }^{\bowtie}$, Dirk Lossnitzer ${ }^{1,2}$, Boris Rudic ${ }^{1,2}$, Erol Tueluemen ${ }^{1,2}$, Johannes Budjan ${ }^{2,3}$, Holger \\ Haubenreisser ${ }^{2,3}$, Thomas Henzler ${ }^{2,3}$, Stefan O. Schoenberg ${ }^{2,3}$, Martin Borggrefe ${ }^{1,2}$, Theano Papavassiliu ${ }^{1,2}$ \\ 1. $1^{\text {st }}$ Department of Medicine Cardiology, University Medical Center Mannheim, Medical Faculty Mannheim, Heidelberg University, Germany \\ 2. DZHK (German Centre for Cardiovascular Research) partner site Mannheim, Germany \\ 3. Institute of Clinical Radiology and Nuclear Medicine, University Medical Center Mannheim, Medical Faculty Mannheim, Heidelberg University, Germany
}

$\triangle$ Corresponding author: Christina Doesch, MD, $1^{\text {st }}$ Department of Medicine Cardiology affiliated at the DZHK (German Centre for Cardiovascular Research) partner site Mannheim, University Medical Center Mannheim, Medical Faculty Mannheim, Heidelberg University, Germany. Theodor-Kutzer-Ufer 1-3, 68167 Mannheim, Germany. Tel.: 0049-621-383-2204, Fax: 0049-621-383-3821, E-Mail: Christina.Doesch@umm.de

() Ivyspring International Publisher. Reproduction is permitted for personal, noncommercial use, provided that the article is in whole, unmodified, and properly cited. See http://ivyspring.com/terms for terms and conditions.

Received: 2015.08.11; Accepted: 2015.10.11; Published: 2016.01.01

\begin{abstract}
Objectives and Background: Atrial fibrillation (AF) is associated with clinical deterioration, stroke and disability in patients with hypertrophic cardiomyopathy (HCM). Therefore, the objective of this study was to evaluated cardiac magnetic resonance (CMR)-derived determinants for the occurrence of $A F$ in patients with HCM.

Methods: 98 Patients with HCM and 30 healthy controls underwent CMR and were followed-up for $6 \pm 3$ years.

Results: 19 (19.4\%) patients presented with AF at initial diagnosis, 19 (19.4\%) developed AF during follow-up and $60(61.2 \%)$ remained in sinus rhythm (SR). Compared to healthy controls, patients with HCM who remained in SR presented with significantly increased left ventricular mass, an elevated left ventricular remodeling index, enlarged left atrial volumes and reduced septal mitral annular plane systolic excursion (MAPSE) compared to healthy controls. Whereas HCM patients who presented with AF at initial diagnosis and those who developed AF during follow-up additionally presented with reduced tricuspid annular plane systolic excursion (TAPSE) and right atrial (RA) dilatation. Receiver-operator curve analysis indicated good predictive performance of TAPSE, RA diameter and septal MAPSE (AUC $0.73,0.69$ and 0.71 , respectively) to detect patients at risk of developing AF.

Conclusion: Reduced MAPSE measurements and enlarged LA volumes seems to be a common feature in patients with HCM, whereas reduced TAPSE and RA dilatation only seem to be altered in patients with history of AF and those developing AF. Therefore, they could serve as easy determinable markers of $\mathrm{AF}$ in patients with $\mathrm{HCM}$.
\end{abstract}

Key words: MAPSE; TAPSE; hypertrophic cardiomyopathy; atrial fibrillation; late gadolinium enhancement; cardiovascular magnetic resonance imaging.

\section{Introduction}

Asymmetric left ventricular hypertrophy in the absence of other causes, such as hypertension or valvular heart disease, is the hallmark of hypertrophic cardiomyopathy (HCM). However, as shown by previous echocardiographic tissue doppler studies [1],
HCM may also involve the right ventricle. Echocardiographically derived mitral and tricuspid annular plane systolic excursions (MAPSE and TAPSE) have been suggested as surrogate markers for longitudinal systolic and diastolic left ventricular (LV) and right 
ventricular (RV) function in several cardiac pathologies $[2,3]$. However, their relevance in HCM is still unclear. Clinically, about one third of patients with HCM develop atrial fibrillation (AF) which is associated with clinical deterioration, stroke, stroke-related death and disability [4]. Therefore, the early identification of patients at risk to develop AF is warrant to allow the timely monitoring and introduction of therapies to protect patients against the consequences of AF.

Thus, the aim of our study was to evaluate cardiac magnetic resonance (CMR)-derived determinants for the occurrence of AF in patients with HCM.

\section{Methods}

\section{Study population}

All patients and volunteers included in this study gave written informed consent and the study was approved by the local ethics commission.

A total of 130 consecutive patients with HCM referred for CMR were enrolled between February 2005 and March 2014 at our department. The population included 60 patients as reported earlier [5]. All patients with HCM were diagnosed based on conventional criteria; left ventricular hypertrophy $\geq 15$ $\mathrm{mm}$ on two-dimensional echocardiography in the absence of another disease that could account for the hypertrophy [6]. Non-obstructive HCM (HNCM) was defined as a pressure gradient $\leq 30 \mathrm{mmHg}$ at rest and after provocation. Patients with a pressure gradient $>30 \mathrm{mmHg}$ at rest or after provocation were classified as obstructive HCM (HOCM).

Of 130 patients with HCM, 32 patients were excluded, due to incomplete clinical or CMR data $(n=22)$, or insufficient CMR image quality $(n=10)$, yielding a total number of 98 finally included in this study (67 men and 31 women; mean age $56 \pm 14$ years).

30 age and sex matched healthy subjects served as controls and satisfied the following criteria: normal physical examination, normal blood pressure $(<130$ $\mathrm{mm} \mathrm{Hg}$ and $<85 \mathrm{~mm} \mathrm{Hg}$ ), normal ECG findings, no history of chest pain or dyspnoea, no diabetes, no hyperlipidemia and normal 2D echocardiography and doppler examination. None of the control subjects was on medication. Exclusion criteria for healthy controls were the presence of signs or symptoms of cardiac diseases, hypertension, diabetes, smoking, or participation in competitive sports.

\section{CMR image acquisition}

All studies were performed using a 1.5 Tesla and 3.0 Tesla whole body MR imaging system (Magnetom Avanto and Skyra, Siemens Medical Systems,
Healthcare Sector, Erlangen, Germany) using a six-element (Avanto) phased-array body coil and a 18-element body matrix coil. Cine images were acquired using a retrospective-gated balanced segmented steady state free precession (trueFISP) sequence in three long-axis views (2-, 3-, and 4-chamber view) and in multiple short-axis views, covering the entire LV from base to apex. Late gadolinium enhancement (LGE) images were obtained 10-15 min after intravenous administration of $0.2 \mathrm{mmol} \mathrm{kg}{ }^{-1}$ Gadoteric acid (Dotarem, Guerbet, Roissy CdG Cedex, France, Germany), using either an inversion recovery turbo Fast Low Angle Shot sequence or a phase-sensitive inversion recovery true fast imaging with steady state precission sequence at the same position as the long and short-axis cine acquisitions in end diastole [7]. The inversion time was adjusted per patient to optimally null signal from normal myocardium typically between 250 and $300 \mathrm{~ms}$.

\section{CMR Image analysis}

Left ventricular mass and volumes as well as right ventricular and left atrial volumes [8] were determined using CMR as previously described. The LV remodelling index (LVRI) was determined as the ratio of LV mass (LV-EDM) to LV end-diastolic volume (LV-EDV). MAPSE measurements were assessed on 4-chamber view cine images. The distance between the basal septal mitral annulus (septal MAPSE) and a reference point outside the heart were measured in end-diastole and end-systole. Septal MAPSE was calculated by subtracting the end-systolic (ESL) from the end-diastolic length (EDL).

To determine TAPSE, the distance between the cutting edge of the tricuspid annulus with the RV free wall and a reference point outside the RV apex were measured in end-diastole ([EDL) and end-systole (ESL). The point outside the RV apex was chosen in extension to the right ventricular apex and had to stay unchanged at end-diastole and end-systole. TAPSE was defined as the difference between EDL and ESL.

\section{Quantification of the severity of mitral regur- gitation}

The mitral regurgitation (MR) volume was calculated through an indirect method [9] and the severity of the MR was quantified using CMR as previously described [9].

Patients with moderate or more than moderate MR were considered as having significant MR[10]. Additionally, MR was determined using color doppler echocardiography. In subjects with a visually assessed moderate MR underwent quantification of the effective regurgitant orifice area (EROA) using a simplified proximal convergence method. Patients 
with $\geq$ moderate $\mathrm{MR}$ and EROA $\geq 30 \mathrm{~mm}^{2}$ were considered as having significant MR [10]. In case of disagreement between the CMR and the echocardiography results quantification of the severity of the mitral regurgitation was done according to the echocardiography results.

\section{Atrial Fibrillation}

AF was documented based on ECG recordings obtained when patients presented with acute onset of symptoms or during follow up visits including 24 hour ambulatory Holter ECG monitoring. AF episodes were classified according to the current guidelines of the European Society of Cardiology [11].

\section{Statistical Analysis}

All data are presented as a mean \pm standard deviation. Continuous parameters were compared using a 2-tailed student's t-test. The Mann-Whitney U test was applied for nonparametric data. Receiver-operator curve analysis (ROC) was used to determine the optimal cut-off value of TAPSE and RAD to discriminate patients at increased risk of AF development.

All results were considered statistically significant when $p<0.05$. Analyses were performed with Statistical Package for Social Sciences (SPSS for windows 14.0, Chicago, IL, USA).

\section{Results}

Table 1 presents CMR characteristics of all 98 patients with HCM compared to 30 healthy controls. Among the 98 patients, 19 (19.4\%) presented with AF at initial diagnosis. Another 19 (19.4\%) developed AF during the follow-up period of $6 \pm 3$ years. $60(61.2 \%)$ remained in SR. Compared to healthy controls, patients with HCM who remained in SR (Table 2) presented with significantly increased indexed left ventricular mass (LV-EDMI, $\mathrm{p}<0.0001$ ), an elevated left ventricular remodeling index (LVRI, $p=0.001)$, increased septal (SWT, $p<0.0001)$ and posterior wall thickness (PWT, $p=0.001$ ), enlarged indexed left atrial volumes $(p<0.0001)$ as well as reduced septal MAPSE measurements $(p=0.003)$.

Left (LV-EF, $p=0.03)$ and right ventricular ejection fraction (RV-EF, $p=0.07)$ were hypercontractile in patients with HCM in SR compared to healthy controls. TAPSE $(p=0.3)$ and RAD $(p=0.3)$ were not different between healthy controls and patients with $\mathrm{HCM}$ in SR.
Table 1: A CMR characteristics of Patients with HCM and healthy controls

\begin{tabular}{|c|c|c|c|}
\hline & $\begin{array}{l}\text { All patients } \\
\text { with HCM } \\
(\mathrm{n}=98)\end{array}$ & $\begin{array}{l}\text { Healthy } \\
\text { Controls } \\
(n=30)\end{array}$ & $\begin{array}{l}\text { p-value all patients } \\
\text { with HCM vs } \\
\text { healthy controls }\end{array}$ \\
\hline Age (yrs) & $56 \pm 14$ & $50 \pm 8$ & 0.06 \\
\hline Male gender $(\mathrm{n} / \%)$ & $67(68)$ & $23(77)$ & 0.5 \\
\hline LV-EF(\%) & $61 \pm 10$ & $59 \pm 4$ & 0.3 \\
\hline LV-EDVI (ml m²) & $77 \pm 20$ & $71 \pm 15$ & 0.2 \\
\hline LV-ESVI $\left(\mathrm{ml} \mathrm{m} \mathrm{m}^{-2}\right)$ & $31 \pm 15$ & $30 \pm 5$ & 0.2 \\
\hline LV-SVI $\left(\mathrm{ml} \mathrm{m} \mathrm{m}^{-2}\right)$ & $46 \pm 12$ & $45 \pm 10$ & 0.3 \\
\hline LV-EDMI (g m-2) & $99 \pm 33$ & $60 \pm 11$ & $<0.0001$ \\
\hline LVRI (g/ml) & $1.3 \pm 0.5$ & $0.9 \pm 0.3$ & 0.0003 \\
\hline LV EDD (mm) & $52 \pm 7$ & $50 \pm 4$ & 0.2 \\
\hline SWT (mm) & $20 \pm 5$ & $9 \pm 2$ & $<0.0001$ \\
\hline PWT (mm) & $10 \pm 3$ & $8 \pm 2$ & 0.0001 \\
\hline indexed LA volume $\left(\mathrm{ml} \mathrm{m}^{-2}\right)$ & $49 \pm 24$ & $22 \pm 3$ & $<0.0001$ \\
\hline Septal MAPSE (cm) & $0.94 \pm 0.37$ & $1.29 \pm 0.27$ & $<0.0001$ \\
\hline TAPSE (cm) & $1.78 \pm 0.58$ & $2.03 \pm 0.17$ & 0.03 \\
\hline RV-EF (\%) & $62 \pm 11$ & $58 \pm 4$ & 0.08 \\
\hline RV-EDVI $\left(\mathrm{ml} \mathrm{m}^{-2}\right)$ & $71 \pm 21$ & $69 \pm 12$ & 0.7 \\
\hline RV-ESVI $\left(\mathrm{ml} \mathrm{m}^{-2}\right)$ & $28 \pm 13$ & $28 \pm 6$ & 0.8 \\
\hline RV-SVI (ml m²) & $43 \pm 11$ & $41 \pm 7$ & 0.3 \\
\hline RVEDD (mm) & $42 \pm 6$ & $41 \pm 7$ & 0.3 \\
\hline RVESD (mm) & $28 \pm 7$ & $27 \pm 6$ & 0.5 \\
\hline $\operatorname{RAD}(\mathrm{mm})$ & $43 \pm 7$ & $40 \pm 4$ & 0.05 \\
\hline $\begin{array}{l}\text { Presence of } \geq \text { moderate mitral } \\
\text { regurgitation }\end{array}$ & $12(12)$ & 0 & - \\
\hline Presence of LGE & $63(64)$ & 0 & - \\
\hline LGE Extent (\%) & $13 \pm 14$ & 0 & - \\
\hline
\end{tabular}

Data are presented as \pm standard deviation. Volumes are indexed to body surface area. $\mathrm{EDD}=\mathrm{end}$ diastolic dimension, $\mathrm{EDMI}=$ end diastolic mass index, $\mathrm{EDVI}=\mathrm{end}$ diastolic volume index, ESVI=end systolic volume index, $\mathrm{LA}=$ left atrial, $\mathrm{LGE}=$ late gadolinium enhancement, $L V=$ left ventricular, $L V R I=$ left ventricular remodeling index, MAPSE $=$ mitral annular plane systolic excursion, $\mathrm{PWT}=$ posterior wall thickness, $\mathrm{SR}=$ sinus rhythm, SWT $=$ septal wall thickness

Table 2: CMR characteristics of Patients with HCM and SR compared to Healthy Controls

\begin{tabular}{|c|c|c|c|}
\hline & $\begin{array}{l}\text { Healthy } \\
\text { Controls } \\
(\mathrm{n}=30)\end{array}$ & $\begin{array}{l}\text { HCM pa- } \\
\text { tients re- } \\
\text { maining in } \\
\text { SR }(n=60)\end{array}$ & $\begin{array}{l}\text { p-value HCM } \\
\text { patients remaining } \\
\text { in SR vs healthy } \\
\text { controls }\end{array}$ \\
\hline Age (yrs) & $50 \pm 8$ & $53 \pm 14$ & 0.4 \\
\hline Male gender $\mathrm{n}(\%)$ & $23(77)$ & $45(75)$ & 0.9 \\
\hline LV-EF (\%) & $59 \pm 4$ & $63 \pm 9$ & 0.03 \\
\hline LV-EDVI $\left(\mathrm{ml} \mathrm{m}^{-2}\right)$ & $71 \pm 15$ & $77 \pm 17$ & 0.1 \\
\hline LV-ESVI $\left(\mathrm{ml} \mathrm{m}^{-2}\right)$ & $30 \pm 5$ & $30 \pm 12$ & 0.3 \\
\hline LV-SVI $\left(\mathrm{ml} \mathrm{m}^{-2}\right)$ & $45 \pm 10$ & $47 \pm 11$ & 0.1 \\
\hline LV-EDMI $\left(\mathrm{g}^{\mathrm{m}} \mathrm{m}^{-2}\right)$ & $60 \pm 11$ & $101 \pm 33$ & $<0.0001$ \\
\hline LVRI (g/ml) & $0.9 \pm 0.3$ & $1.3 \pm 0.5$ & 0.001 \\
\hline LV EDD (mm) & $50 \pm 4$ & $53 \pm 6$ & 0.05 \\
\hline SWT (mm) & $9 \pm 2$ & $20 \pm 6$ & $<0.0001$ \\
\hline PWT (mm) & $8 \pm 2$ & $10 \pm 3$ & 0.001 \\
\hline Indexed LA volume $\left(\mathrm{ml} \mathrm{m}^{-2}\right)$ & $22 \pm 3$ & $44 \pm 21$ & $<0.0001$ \\
\hline Septal MAPSE (cm) & $1.29 \pm 0.27$ & $1.04 \pm 0.38$ & 0.003 \\
\hline TAPSE & $2.03 \pm 0.17$ & $1.91 \pm 0.59$ & 0.3 \\
\hline RV-EF (\%) & $58 \pm 4$ & $63 \pm 11$ & 0.07 \\
\hline RV-EDVI $\left(\mathrm{ml} \mathrm{m}^{-2}\right)$ & $69 \pm 12$ & $71 \pm 23$ & 0.5 \\
\hline RV-ESVI $\left(\mathrm{ml} \mathrm{m}^{-2}\right)$ & $28 \pm 6$ & $27 \pm 13$ & 0.7 \\
\hline RV-SVI (ml m²) & $41 \pm 7$ & $44 \pm 10$ & 0.2 \\
\hline RVEDD (mm) & $41 \pm 7$ & $42 \pm 6$ & 0.2 \\
\hline RVESD (mm) & $27 \pm 6$ & $28 \pm 7$ & 0.5 \\
\hline RAD (mm) & $40 \pm 4$ & $42 \pm 7$ & 0.3 \\
\hline $\begin{array}{l}\text { Presence of } \geq \text { moderate mitral } \\
\text { regurgitation } n(\%)\end{array}$ & 0 & $6(10)$ & - \\
\hline Presence of LGE & 0 & $36(60)$ & - \\
\hline LGE Extent (\%) & 0 & $11 \pm 12$ & - \\
\hline \multicolumn{4}{|c|}{$\begin{array}{l}\text { Data are presented as } \pm \text { standard deviation. Volumes are indexed to body surface area. } \\
\text { EDD=end diastolic dimension, EDMI= end diastolic mass index, EDVI=end diastolic } \\
\text { volume index, ESVI=end systolic volume index, } \mathrm{LA}=\text { left atrial, } \mathrm{LGE}=\text { late gadolinium } \\
\text { enhancement, } \mathrm{LV}=\text { left ventricular, } \mathrm{LVRI}=\text { left ventricular remodeling index, MAPSE = } \\
\text { mitral annular plane systolic excursion, PWT=posterior wall thickness, SWT=septal wall } \\
\text { thickness }\end{array}$} \\
\hline
\end{tabular}


Looking at the LGE extent \% in HCM patients who remained in SR $(11 \pm 12 \%)$ compared to those who presented with AF at initial diagnosis $(16 \pm 16 \%)$ or during follow-up $(17 \pm 18 \%)$ showed a trend towards more LGE $(16 \pm 16 \%, \mathrm{p}=0.2$ and $17 \pm 18 \%, \mathrm{p}=0.1$, respectively). In relation to patients with HCM in SR, those who presented with AF at initial diagnosis (Table 3) were significantly older $(p=0.01)$, revealed significantly elevated indexed LA volumes $(p=0.004)$, significantly reduced septal MAPSE $(p=0.01$, Figure 1 D, $\mathrm{E})$, significantly reduced TAPSE $(\mathrm{p}=0.03)$ and dilated RA (RAD, $p=0.01$, Figure $1 \mathrm{~F}$ ).

Comparing patients with HCM in SR (Figure 1 A-C) with those who developed AF during follow-up (Figure 1 G-I), the latter presented with significantly reduced septal MAPSE $(p=0.01)$, significantly diminished TAPSE $(p=0.02)$ and significantly higher RAD $(\mathrm{p}=0.01)$.

ROC analysis (Figure 2) indicated good predictive performance of TAPSE, RAD and septal MAPSE (AUC $0.73,0.69$ and 0.71 , respectively). A cut-off at 1.7 for TAPSE had a sensitivity of $79 \%$ and a specificity of $70 \%$ to predict the occurrence of AF during follow-up. With respect to RAD, a cut-off of $47.5 \mathrm{~mm}$ for RAD revealed AF during follow-up with a sensitivity of $74 \%$ and a specificity of $77 \%$. With a cut-off at $0.9 \mathrm{~cm}$ for septal MAPSE had a sensitivity of $90 \%$ and a specificity of $62 \%$ to predict the occurrence of AF during follow-up.

\section{Discussion}

In patients with $\mathrm{HCM}$, despite normal radial LV and RV function, longitudinal contraction was significantly impaired to healthy controls. Furthermore, irrespective of the presence or incidence of $\mathrm{AF}, \mathrm{LA}$ was enlarged and MAPSE measurements were diminished in all patients with HCM compared to healthy controls. However, HCM patients with history of AF and those at risk to develop AF both additionally presented with RA dilatation and reduced TAPSE.

Thus, variable degrees of LA dilatation and reduced septal MAPSE seem to be a common feature in all patients with HCM whereas enlarged RA volumes and reduced TAPSE only seem to be present in patients with history and at risk to develop AF. Therefore RAD and TAPSE measurements could serve as easy to determine predictors of AF.
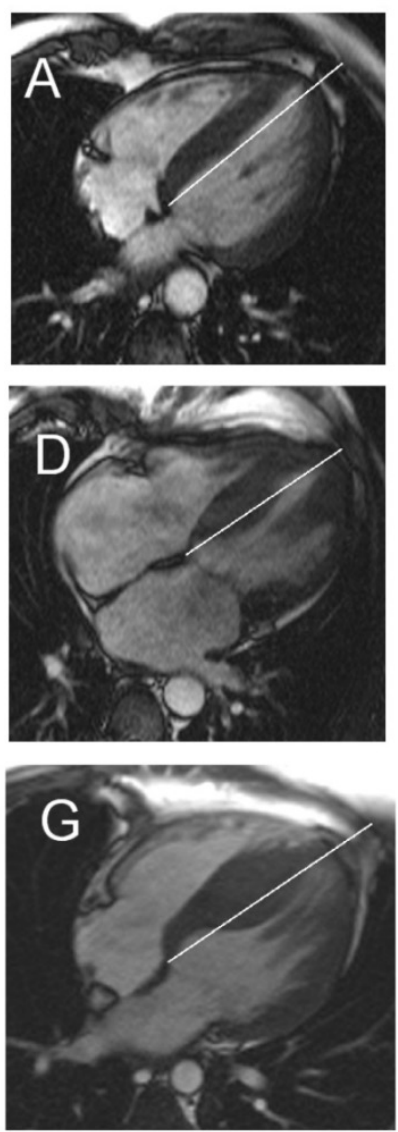
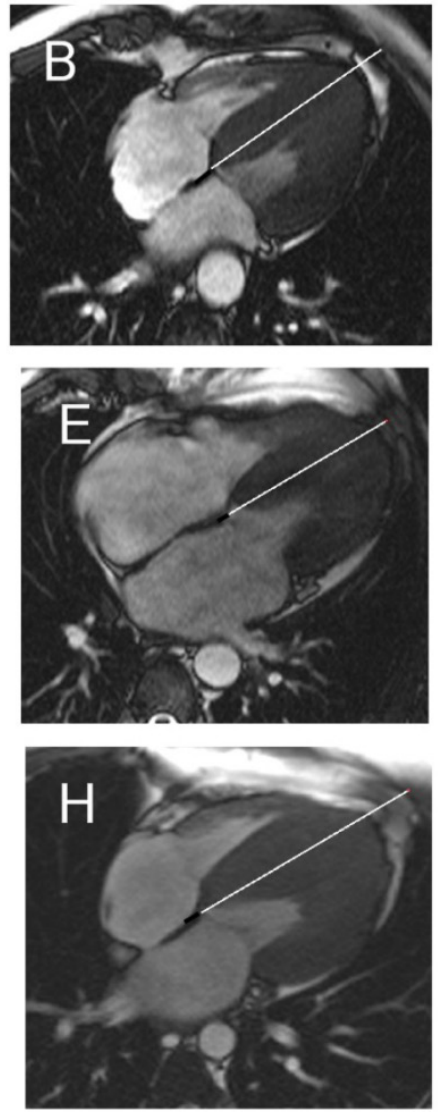
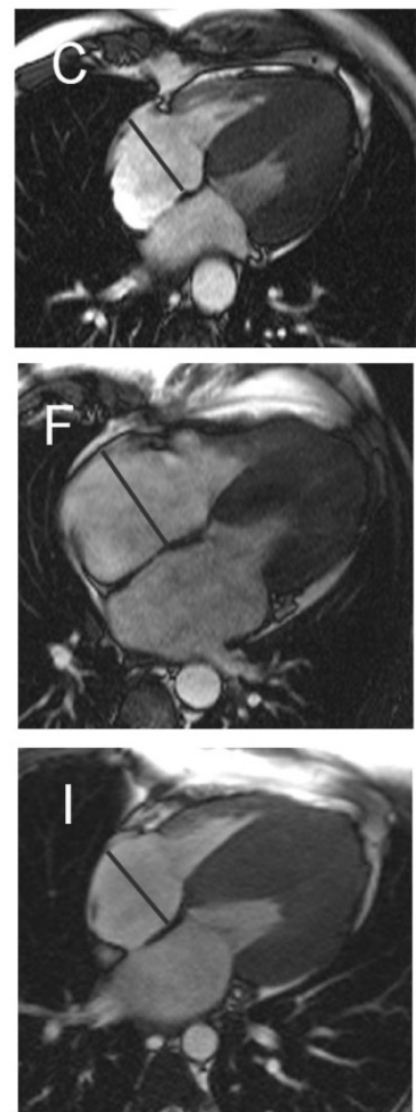

Figure 1: Representative Figure. In patients with HCM remaining in sinus rhythm (A-C), in those with atrial fibrillation at intial diagnosis (D-F) and those developing atrial fibrillation during follow-up (G-I), septal MAPSE (black line $B, E, H)$ was calculated by subtracting the end-systolic (B, E, H) from the end-diastolic length (A, D, G) and the RAD (C, F, I) was determined. 
A

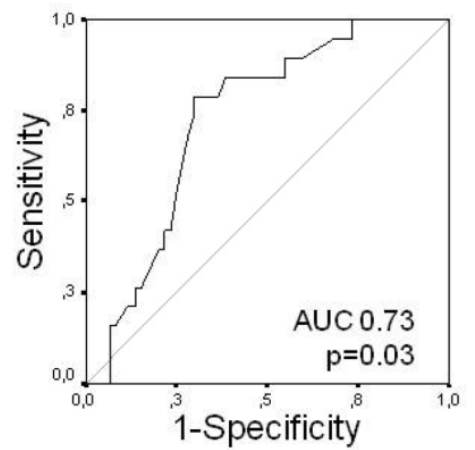

B

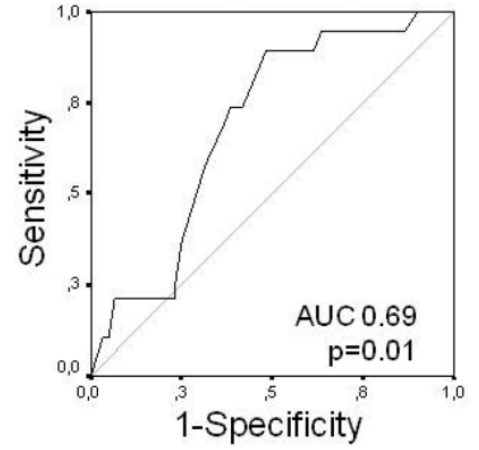

C

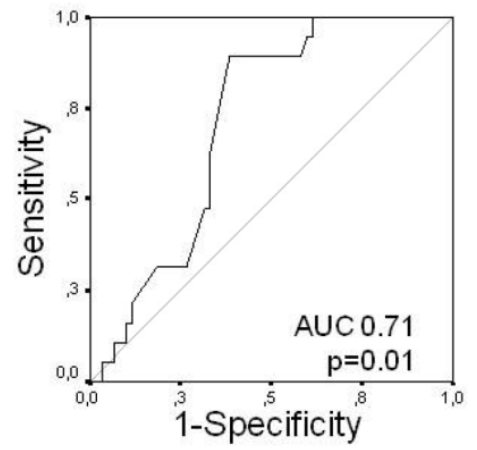

Figure 2 Receiver operating characteristic (ROC) curves analysis. ROC curves for TAPSE (Panel A), RAD (Panel B) and septal MAPSE (Panel C) to predict the occurrence of atrial fibrillation during follow-up.

So far, the pathophysiological mechanisms that predispose to $\mathrm{AF}$ in patients with $\mathrm{HCM}$ are poorly understood. It is known that LA size [12], LA volume $[5,13]$ and LA function [14] as well as advanced age [14] are independently associated with AF in patients with HCM. In line with these results, in the present study, patients with HCM who presented with AF at initial diagnosis showed the most pronounced LA dilatation. Patients with HCM who remained in SR and those who developed AF during follow-up both also revealed significantly dilated LA compared to healthy controls. However, LA volumes were comparable between patients who remained in SR and those who developed AF during follow-up. Thus, LA dilatation was not considered to be able to discriminate those at risk to develop AF from those remaining in SR.
Table 3: CMR characteristics of Patients with HCM with regard to AF

\begin{tabular}{|c|c|c|c|c|c|}
\hline & $\begin{array}{l}\mathrm{HCM} \\
\text { patients } \\
\text { remaining } \\
\text { in SR } \\
(\mathrm{n}=60)\end{array}$ & $\begin{array}{l}\text { Patients } \\
\text { with AF at } \\
\text { initial } \\
\text { diagnosis } \\
(\mathrm{n}=19)\end{array}$ & $\begin{array}{l}\text { p-value } \\
\text { HCM pa- } \\
\text { tients re- } \\
\text { maining in } \\
\text { SR vs HCM } \\
\text { patients with } \\
\text { AF at initial } \\
\text { diagnosis }\end{array}$ & $\begin{array}{l}\text { Patients } \\
\text { with AF } \\
\text { during } \\
\text { fol- } \\
\text { low-up } \\
(\mathrm{n}=19)\end{array}$ & $\begin{array}{l}\text { p-value } \\
\text { HCM pa- } \\
\text { tients re- } \\
\text { maining in } \\
\text { SR vs HCM } \\
\text { patients with } \\
\text { AF during } \\
\text { follow-up }\end{array}$ \\
\hline Age (yrs) & $53 \pm 14$ & $63 \pm 11$ & 0.01 & $59 \pm 11$ & 0.1 \\
\hline $\begin{array}{l}\text { Male gender } \\
\mathrm{n}(\%)\end{array}$ & $45(75)$ & $12(63)$ & 0.2 & $10(53)$ & 0.9 \\
\hline LV-EF (\%) & $63 \pm 9$ & $59 \pm 11$ & 0.1 & $58 \pm 10$ & 0.1 \\
\hline LV-EDVI (ml m²-2) & $77 \pm 17$ & $78 \pm 27$ & 0.8 & $75 \pm 22$ & 0.6 \\
\hline LV-ESVI (ml m²-2) & $30 \pm 12$ & $35 \pm 23$ & 0.3 & $32 \pm 18$ & 0.6 \\
\hline LV-SVI (ml m-2) & $47 \pm 11$ & $48 \pm 15$ & 0.9 & $41 \pm 13$ & 0.1 \\
\hline LV-EDMI $\left(\mathrm{g} \mathrm{m}^{-2}\right)$ & $101 \pm 33$ & $104 \pm 41$ & 0.8 & $90 \pm 24$ & 0.2 \\
\hline LVRI (g/ml) & $1.3 \pm 0.5$ & $1.4 \pm 0.6$ & 0.6 & $1.3 \pm 0.3$ & 0.5 \\
\hline LV EDD (mm) & $53 \pm 6$ & $51 \pm 8$ & 0.5 & $51 \pm 7$ & 0.2 \\
\hline SWT (mm) & $20 \pm 6$ & $19 \pm 4$ & 0.5 & $20 \pm 5$ & 0.8 \\
\hline PWT (mm) & $10 \pm 3$ & $10 \pm 2$ & 0.7 & $10 \pm 2$ & 0.8 \\
\hline $\begin{array}{l}\text { Indexed LA volume } \\
\left(\mathrm{ml} \mathrm{m}^{-2}\right)\end{array}$ & $44 \pm 21$ & $62 \pm 26$ & 0.004 & $53 \pm 28$ & 0.1 \\
\hline Septal MAPSE (cm) & $1.04 \pm 0.38$ & $0.76 \pm 0.38$ & 0.01 & $\begin{array}{l}0.80 \pm 0.1 \\
9\end{array}$ & 0.01 \\
\hline TAPSE & $1.91 \pm 0.59$ & $1.57 \pm 0.59$ & 0.03 & $\begin{array}{l}1.60 \pm 0.4 \\
3\end{array}$ & 0.02 \\
\hline RV-EF (\%) & $63 \pm 11$ & $61 \pm 10$ & 0.4 & $62 \pm 10$ & 0.8 \\
\hline RV-EDVI (ml m²-2) & $71 \pm 23$ & $70 \pm 17$ & 0.8 & $68 \pm 19$ & 0.6 \\
\hline RV-ESVI (ml m²) & $27 \pm 13$ & $28 \pm 12$ & 0.9 & $28 \pm 14$ & 0.8 \\
\hline RV-SVI (ml m-2) & $44 \pm 10$ & $42 \pm 11$ & 0.5 & $40 \pm 11$ & 0.2 \\
\hline RVEDD (mm) & $42 \pm 6$ & $41 \pm 6$ & 0.2 & $42 \pm 6$ & 0.9 \\
\hline RVESD (mm) & $28 \pm 7$ & $27 \pm 6$ & 0.7 & $29 \pm 7$ & 0.8 \\
\hline RAD (mm) & $42 \pm 7$ & $47 \pm 6$ & 0.01 & $46 \pm 6$ & 0.01 \\
\hline $\begin{array}{l}\text { Presence of } \geq \text { mod- } \\
\text { erate mitral regur- } \\
\text { gitation } n(\%)\end{array}$ & $6(10)$ & $4(21)$ & 0.4 & $2(22)$ & 0.9 \\
\hline Presence of LGE & $36(60)$ & $14(74)$ & 0.6 & $13(68)$ & 0.2 \\
\hline LGE Extent (\%) & $11 \pm 12$ & $16 \pm 16$ & 0.2 & $17 \pm 18$ & 0.1 \\
\hline
\end{tabular}

Another factor that has been related to AF development in patients with HCM is LGE. In an autopsy study by Yamaji et al. [15], LV fibrosis was detected histologically in patients with HCM and AF more frequently. This was in line with a previous study of our group [5] that also found significantly more LGE in HCM patients with AF compared to those without AF. In the present study, patients with AF or those who developed AF during follow-up also showed a trend towards more LGE compared to those who remained in SR.

It is known that the hypertrophic process in HCM may also affect the right ventricle [16] In patients with HCM and a concomitant RV involvement, McKenna WJ et al. [16] reported a significant association between RV involvement and severity of symptoms. Besides, they reported an increased incidence of ventricular tachycardias and supraventricular ar- 
rhythmias in these patients. The structure and motion of the RV has been studied to a lesser extent, but movement of the free wall is primarily longitudinal with an element of radial contraction towards the apex and anterior section [17]. In patients with HCM two dimensional strain echocardiography has shown that despite preserved radial function, longitudinal contraction might be reduced representing the early phase of disease [18]. Longitudinal RV function can be estimated using the semi-quantitative TAPSE measurement [2]. In our study, patients who presented with AF at initial diagnosis or during follow-up showed significantly reduced TAPSE compared to healthy controls as well as to patients who remained in SR. Moreover, patients with HCM who presented with AF or develop AF during follow-up had significantly enlarged right atria compared to those patients who remained in SR or healthy controls. This finding is consistent with previous reports describing an association between dilation of the right atrium and higher incidence of AF [19]. TAPSE measurements and RAD were comparable among patients with HCM who remained in SR and healthy controls.

$\mathrm{AF}$ is the most common arrhythmia in patients with HCM [20] and develops in at least $20 \%$ of patients. However, the true incidence of AF is probably higher. In our study population the prevalence and incidence of AF were both $19.4 \%$. Since even paroxysmal episodes of AF constitute a thromboembolic risk and may cause rapid clinical deterioration in patients with HCM, the occurrence of AF has shown to predict morbidity and mortality [4], emphasizing the paramount clinical importance to identify predictors of $\mathrm{AF}$.

\section{Clinical implication}

In our study reduced TAPSE and increased RAD showed good predictive performance with regard to the development of AF. Due to the association between AF and increased morbidity and mortality in patients with HCM [4], oral anticoagulation should be prescribed early in this condition. In the presence of reduced longitudinal RV contraction and right atrial dilatation determined by CMR, patients with $\mathrm{HCM}$ should be screened for symptoms of AF. Serial 12-lead ECGs and Holter monitoring should be recommended to detect AF as early as possible.

\section{Conclusion}

Reduced MAPSE measurements and LA dilation seems to be a common feature in patients with HCM, whereas reduced TAPSE and RA dilatation only seem to be altered in patients with history of AF and those developing AF. Therefore, they could serve as easy determinable markers of $\mathrm{AF}$ in patients with $\mathrm{HCM}$ indicating patients in whom early screening for AF might be beneficial.

\section{Acknowledgements}

This study was supported by the DZHK (German Centre for Cardiovascular Research) and by the BMBF (German Ministry of Education and Research).

\section{Abbreviations}

AF: atrial fibrillation

CMR: cardiovascular magnetic resonance imaging

ECG: Electrocardiogram

HCM: hypertrophic cardiomyopathy

HNCM: hypertrophic non-obstructive cardiomyopathy

HOCM: hypertrophic obstructive cardiomyopathy

LA: left atrium

LGE: late gadolinium enhancement

$\mathrm{LV}$ : left ventricular

MAPSE: mitral annular plane systolic excursion

TAPSE: tricuspid annular plane systolic excursion

\section{Competing Interests}

The authors have declared that no competing interest exists.

\section{References}

1. Morner $S$, Lindqvist $P$, Waldenstrom $A$, et al. Right ventricular dysfunction in hypertrophic cardiomyopathy as evidenced by the myocardial performance index. Int J Cardiol. 2008; 124: 57-63.

2. Kaul S, Tei C, Hopkins JM, et al. Assessment of right ventricular function using two-dimensional echocardiography. Am Heart J. 1984; 107: 526-531.

3. Wenzelburger FW, Tan YT, Choudhary FJ, et al. Mitral annular plane systolic excursion on exercise: a simple diagnostic tool for heart failure with preserved ejection fraction. Eur J Heart Fail. 2011; 13: 953-960.

4. Olivotto I, Cecchi F, Casey SA, et al. Impact of atrial fibrillation on the clinical course of hypertrophic cardiomyopathy. Circulation. 2001; 104: 2517-2524.

5. Papavassiliu T, Germans T, Fluchter S, et al. CMR findings in patients with hypertrophic cardiomyopathy and atrial fibrillation. J Cardiovasc Magn Reson. 2009; 11: 34

6. Maron BJ, Towbin JA, Thiene G, et al. Contemporary definitions and classification of the cardiomyopathies: an American Heart Association Scientific Statement from the Council on Clinical Cardiology, Heart Failure and Transplantation Committee; Quality of Care and Outcomes Research and Functional Genomics and Translational Biology Interdisciplinary Working Groups; and Council on Epidemiology and Prevention. Circulation. 2006; 113: 1807-1816

7. Simonetti OP, Kim RJ, Fieno DS, et al. An improved MR imaging technique for the visualization of myocardial infarction. Radiology. 2001; 218: 215-223.

8. Sievers B, Kirchberg S, Addo M, et al. Assessment of left atrial volumes in sinus rhythm and atrial fibrillation using the biplane area-length method and cardiovascular magnetic resonance imaging with TrueFISP. J Cardiovasc Magn Reson. 2004; 6: 855-863.

9. Gelfand EV, Hughes S, Hauser TH, et al. Severity of mitral and aortic regurgitation as assessed by cardiovascular magnetic resonance: optimizing correlation with Doppler echocardiography. J Cardiovasc Magn Reson. 2006; 8: 503-507.

10. Bruch C, Stypmann J, Gradaus R, et al. Usefulness of tissue Doppler imaging for estimation of filling pressures in patients with primary or secondary pure mitral regurgitation. Am J Cardiol. 2004; 93: 324-328.

11. Camm AJ, Kirchhof P, Lip GY, et al. Guidelines for the management of atrial fibrillation: the Task Force for the Management of Atrial Fibrillation of the European Society of Cardiology (ESC). Eur Heart J. 2010; 31: 2369-2429.

12. Cecchi F, Olivotto I, Montereggi A, et al. Hypertrophic cardiomyopathy in Tuscany: clinical course and outcome in an unselected regional population. J Am Coll Cardiol. 1995; 26: 1529-1536.

13. Losi MA, Betocchi S, Aversa M, et al. Determinants of atrial fibrillation development in patients with hypertrophic cardiomyopathy. Am J Cardiol. 2004; 94: 895-900. 
14. Maron BJ, Haas TS, Maron MS, et al. Left atrial remodeling in hypertrophic cardiomyopathy and susceptibility markers for atrial fibrillation identified by cardiovascular magnetic resonance. Am J Cardiol. 2014; 113: 1394-1400.

15. Yamaji K, Fujimoto S, Yutani C, et al. Does the progression of myocardial fibrosis lead to atrial fibrillation in patients with hypertrophic cardiomyopathy? Cardiovasc Pathol. 2001; 10: 297-303.

16. McKenna WJ, Kleinebenne A, Nihoyannopoulos P, et al. Echocardiographic measurement of right ventricular wall thickness in hypertrophic cardiomyopathy: relation to clinical and prognostic features. J Am Coll Cardiol. 1988; 11: 351-358.

17. Scott AD, Keegan J, and Firmin DN. Motion in cardiovascular MR imaging. Radiology. 2009; 250: 331-351.

18. Serri $\mathrm{K}$, Reant $\mathrm{P}$, Lafitte $\mathrm{M}$, et al. Global and regional myocardial function quantification by two-dimensional strain: application in hypertrophic cardiomyopathy. J Am Coll Cardiol. 2006; 47: 1175-1181.

19. Levy S, Breithardt G, Campbell RW, et al. Atrial fibrillation: current knowledge and recommendations for management. Working Group on $\mathrm{Ar}-$ rhythmias of the European Society of Cardiology. Eur Heart J. 1998; 19: 1294-1320.

20. Maron BJ, McKenna WJ, Danielson GK, et al. American College of Cardiology/European Society of Cardiology clinical expert consensus document on hypertrophic cardiomyopathy. A report of the American College of Cardiology Foundation Task Force on Clinical Expert Consensus Documents and the European Society of Cardiology Committee for Practice Guidelines. J Am Coll Cardiol. 2003; 42: 1687-1713. 